\title{
Interdigitated Electrophotocatalytic Cell for Water Purification
}

\author{
Guy Shemer and Yaron Paz \\ Department of Chemical Engineering and the Grand Water Research Institute, Technion, Haifa 32000, Israel \\ Correspondence should be addressed to Yaron Paz, paz@tx.technion.ac.il \\ Received 30 January 2011; Accepted 13 April 2011 \\ Academic Editor: Peter Robertson
}

Copyright ( $) 2011$ G. Shemer and Y. Paz. This is an open access article distributed under the Creative Commons Attribution License, which permits unrestricted use, distribution, and reproduction in any medium, provided the original work is properly cited.

The preparation, characterization, and performance of an electrophotocatalytic cell, made of low-cost, planar interdigitated electrodes is reported hereby. The operation of the cell under small positive bias was demonstrated by photocatalytically degrading the dye rhodamine $6 \mathrm{G}$ in solution as well as by monitoring the degradation of self-assembled monolayer chemisorbed on the $\mathrm{TiO}_{2}$ electrode. Results point out to the importance of activated oxygen species formed in the process and suggest that the short distance between the two electrodes provides a way to utilize the activated oxygen species formed at the negatively biased electrode.

\section{Introduction}

Photocatalytic degradation of pollutants in water and air attracts increasing attention. In this context, titanium dioxide is regarded as the photocatalyst of choice, being inexpensive, nontoxic, and highly efficient if compared with other photocatalysts. The general scheme for the photocatalytic destruction of organics involves excitation with suprabandgap photons and migration of the electronhole pairs to the surface of the photocatalyst, where the holes may be trapped by $\mathrm{H}_{2} \mathrm{O}$ or $\mathrm{OH}^{-}$adsorbed at the surface, thus forming hydroxyl radicals [1]. In parallel, the electrons reduce adsorbed oxygen [2] or are trapped in oxygen vacancies deep traps [3]. Most organic compounds are degraded oxidatively by the hydroxyl radicals, thus producing short-live organic radicals that undergo secondary reactions to form stable molecules, such as $\mathrm{CO}_{2}$ and water [4]. Nevertheless, it was shown that halo-organics, such as 2-bromo-2-chloro-1,1,1-trifluoroethane [5], as well as highly toxic heavy metals ions, such as $\mathrm{Cr}$ (VI) [6], could be degraded reductively by photoinduced electrons.

For photocatalysis to take place, efficient separation of the photoinduced charge carriers is required. Accordingly, coupling to electron sinks, such as platinum or gold nanoislands $[7,8]$ or even carbon nanotubes [9], was found to be quite benevolent in this aspect. Another way to promote charge separation is to anodically bias a titanium dioxidecovered electrode, while maintaining a negatively biased $\mathrm{Pt}$ electrode in the solution $[10,11]$. In that case, the photocatalyst has to be attached to the positive electrode. Fixing the photocatalyst to the electrode solves the problem of separating between the photocatalyst particles and the purified solution once the process is over. Nevertheless, one needs to take into account a reduction in the active surface area of the photocatalyst.

The importance of oxygen in electrochemically assisted photocatalysis was demonstrated in the photocatalytic degradation of 4-chlorophenol [12]. Here, the electrodes were separated by a glass frit, and oxygen was bubbled to the system next to the negatively biased electrode. By comparing between reaction rates and distribution of intermediates with and without the glass frit it was concluded that activated oxygen species formed at the negatively biased electrode did not contribute significantly to the photocatalytic degradation scheme of 4-chlorophenol.

Anodically biasing the $\mathrm{TiO}_{2}$ electrode not only may affect the recombination rate of the photogenerated charge carriers but also may influence the adsorption of the contaminants, many of which are charged, in particular under nonneutral $\mathrm{pH}$. Using chemisorbed self-assembled monolayers attached to surfaces may provide a tool to differentiate between the effect of adsorption and that of reaction. Indeed, 
self-assembled monolayers anchored at the vicinity of titanium dioxide served in the past to study the phenomenon of remote photocatalytic degradation $[13,14]$ and to construct molecular recognition sites, for obtaining selective photocatalysis [15].

In this paper, we report on the preparation, characterization, and performance of a new electrophotocatalytic cell, with both anchored and nonanchored model pollutants. Unlike other biased photocatalytic cells, the cell described below has a planar interdigitated layout that can be mass produced very easily. Since the water flow is parallel to the electrodes' surface, the pressure drop is minimal. Moreover, the cell described below points out to the importance of activated oxygen species and suggests that the short distance between the two electrodes provides a way to utilize the activated oxygen species formed at the negatively biased electrode.

\section{Experimental}

Figure 1 presents the sidewise and top-view schematics of a typical device, having an overall interdigitated part of $0.5 \mathrm{~cm}$ $\times 1.5 \mathrm{~cm}$. The devices were prepared on $2^{\prime \prime}$ silicon wafers onto which a thick $(2 \mu \mathrm{m})$ insulating layer of silicon dioxide was grown by plasma-enhanced chemical vapor deposition. Thin $(7 \mathrm{~nm})$ titanium layer, serving as an adhesion promoter and a thicker $(100 \mathrm{~nm})$ platinum layer were then deposited by evaporation on a prepatterned positive photoresist (AZ1818, Shipley), which has been developed by a standard developer (DEV 326, Shipley). The metals were patterned by a "lift-off" process, that is, by removing the prepatterned photoresist, leaving an interdigitated platinum skeleton consisting of both electrodes (marked in Figure 1 as Pt1 and Pt2), with their pads. The next step consisted of overcoating the whole wafer by a $80 \mathrm{~nm}$ thick layer of titanium dioxide. This layer was introduced by spin coating of an organotitanate precursor, following by calcination. Details of this step were given elsewhere, as this type of $\mathrm{TiO}_{2}$ film was used by us in the past in the developing of self-cleaning glass [16]. Patterning of the titanium dioxide film was done by protecting the appropriate areas by a patterned photoresist (AZ1818, Shipley, developed by DEV326 developer and postbaked at $120^{\circ} \mathrm{C}$ for $10 \mathrm{~min}$.) and etching the exposed $\mathrm{TiO}_{2}$ areas by reactive ion etching (RIE) using $\mathrm{CHF}_{3}$ plasma as an etchant. The RIE step was followed by stripping the photoresist and descumming the wafer with oxygen plasma. Care was taken to make the $\mathrm{TiO}_{2}$ fingers $5 \mu \mathrm{m}$ wider than the underlying Pt2 back-contact electrodes in order to prevent dark current leakage.

Different sets of interdigitated electrodes were prepared by altering the layout of lithographic masks printed by a commercial printer on transparent plastic sheets. Masks with different finger widths $(100,50$, and $25 \mu \mathrm{m})$ and different interfinger distances $(100,50$, and $25 \mu \mathrm{m})$ were prepared, all of which had finger length of $5 \mathrm{~mm}$ and pad length of $15 \mathrm{~mm}$. Devices with equal finger spacing of 25 to $100 \mu \mathrm{m}$ led to 200 to 50 pairs of fingers and insulating gaps, respectively. The total area of the titanium dioxide fingers in each wafer

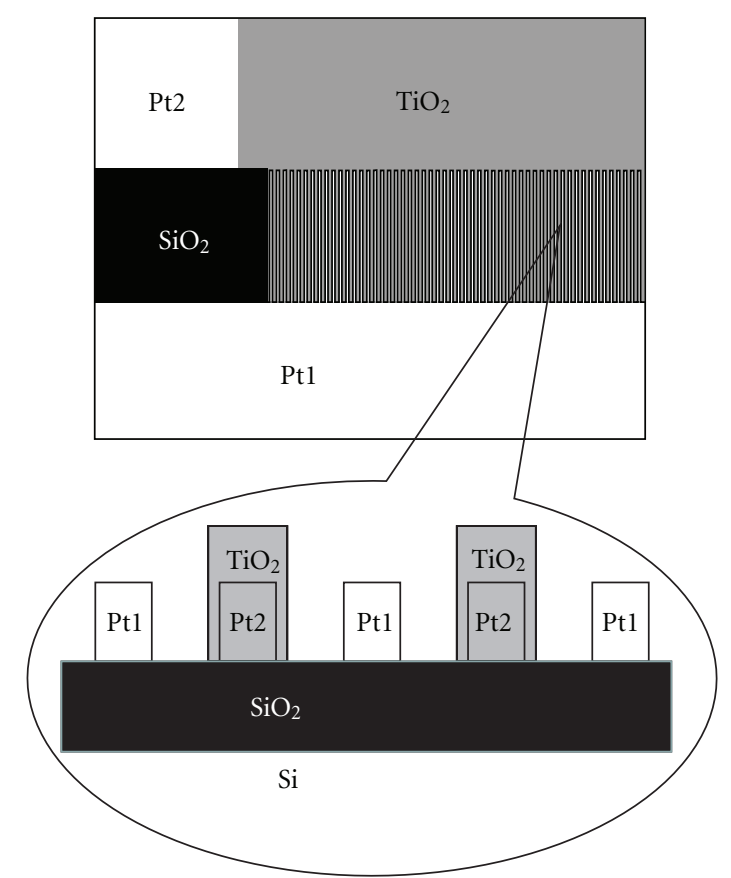

FIGURE 1: Schematics of an interdigitated device (not to scale). The titanium dioxide part is in gray, the insulating $\left(\mathrm{SiO}_{2}\right)$ area is in black, and the platinum electrodes and connectors are in white.

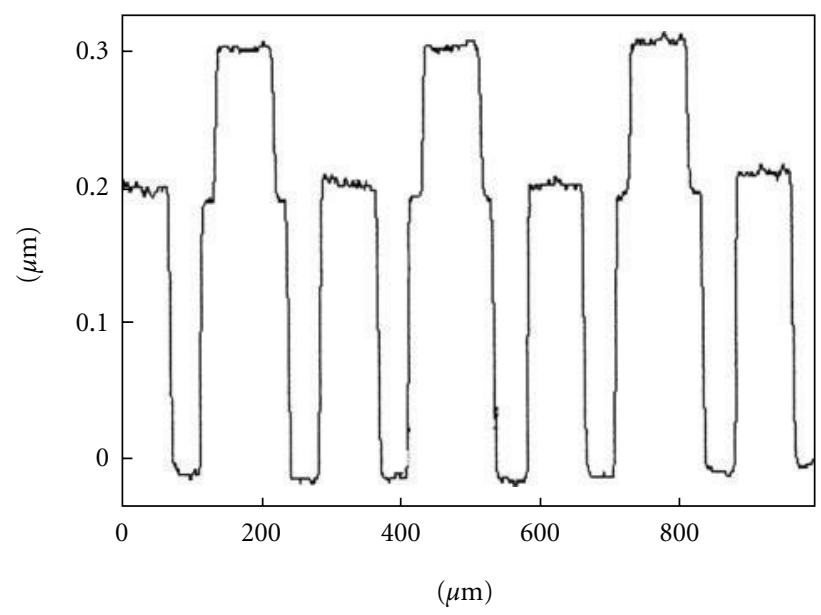

Figure 2: Depth profile of an interdigitated electrode structure.

having the same width of $\mathrm{TiO}_{2}, \mathrm{Pt}$, and $\mathrm{SiO}_{2}$ was $0.1875 \mathrm{~cm}^{2}$. Figure 2 shows a depth profile parallel to the direction of the interdigitated electrodes, for a structure having $\mathrm{TiO}_{2}$ electrodes, $\mathrm{SiO}_{2}$ gaps, and platinum electrodes of nominal widths of $100 \mu \mathrm{m}, 50 \mu \mathrm{m}$, and $100 \mu \mathrm{m}$, respectively. The thickness of the $\mathrm{TiO}_{2}$ layer is approximately $80 \mathrm{~nm}$. To assure electrical isolation between the two types of electrodes, a $0.1 \mu \mathrm{m}$ overetching into the thick silica layer was performed (noticed in the figure by trench depth of $0.2 \mu \mathrm{m}$, some $0.1 \mu \mathrm{m}$ deeper than the thickness of the metal layer).

The interdigitated electrodes were wired using silver paint adhesive. The contacts and the rest of the platinum and titania pads were then overcoated with epoxy glue, such that 
the titanium dioxide working area was limited to that of the interdigitated electrodes. The interdigitated electrodes were inspected routinely by optical microscopy and profilometry at all stages of preparation and were also characterized by XPS and AES during process development.

\subsection{Photocatalysis Measurements}

2.1.1. Degradation of Rhodamine 6G. The effect of biasing the electrodes on the photocatalytic activity was studied by monitoring the photocatalytic degradation of the dye stuff rhodamine 6G. Experiments took place in a flask, containing $40 \mathrm{~mL}$ of aqueous solution of $2.7 \mu \mathrm{M}$ R6G and one wafer $\left(\mathrm{TiO}_{2}\right.$ active area $\left.=0.19 \mathrm{~cm}^{2}\right)$. During experiments, the wafers were exposed to $0.44 \mathrm{~mW} / \mathrm{cm}^{2}$ of UV-A light. The photodegradation kinetics was deduced by following the $528 \mathrm{~nm}$ peak R6G, as measured by a UV-Vis spectrophotometer (Lambda40, Perkin Elmer).

2.1.2. Degradation of a Monolayer of Octyltrichlorosilane. Kinetic measurements of the effect of bias on the photocatalytic degradation of a chemisorbed self-assembled monolayer of octyltrichlorosilane (OCTS, $\left.\mathrm{CH}_{3}\left(\mathrm{CH}_{2}\right)_{7} \mathrm{SiCl}_{3}\right)$ were performed in air. The self-assembled monolayers were chemisorbed on the oxides $\left(\mathrm{SiO}_{2}\right.$ and $\left.\mathrm{TiO}_{2}\right)$ surfaces of the interdigitated electrodes (but not on Pt). More details on the procedure, used by us before for the study of remote degradation effects, can be found elsewhere [14]. During measurements, the coated interdigitated electrodes were exposed to UVA light $\left(0.61 \mathrm{~mW} / \mathrm{cm}^{2}\right)$ under specific bias. The wafers were measured by FTIR following specific exposure times. Kinetics was deduced based on integrating the envelope of the $\mathrm{C}-\mathrm{H}$ stretch peaks at $2800-3000 \mathrm{~cm}^{-1}$.

\section{Results and Discussion}

Figure 3 presents the rhodamine $6 \mathrm{G}$ spectra during its photocatalytic degradation under open-circuit conditions (a) and under a positive bias of $400 \mathrm{mV}$ applied to the titanium electrode (b). A decrease in the R6G signal as a function of exposure time is observed in both cases. Faster kinetics in the biased case is clearly observed. In both cases, this decrease is not accompanied with blue or red shifting of the peak. Nevertheless, at long exposure times, a blue shifting can be recognized in the biased case. It is noteworthy that no degradation was observed in this dye (unlike other dyes) upon exposure in the absence of $\mathrm{TiO}_{2}$.

It has been reported that the photodegradation of the rhodamine family of dyes (mainly rhodamine 6G and rhodamine $\mathrm{B}(\mathrm{RB})$ ) may begin either by $\mathrm{N}$-dealkylation $(\mathrm{N}-$ deethylation in $\mathrm{RB}$ and $\mathrm{N}$-deesterization in $\mathrm{R} 6 \mathrm{G}$ ) or by the degradation of the chromophore [17]. Photocatalytically induced spectral blue shifting is attributed to N-dealkylation, whereas chromophore degradation is manifested by reduction in the peak intensity [18]. N-dealkylation was found in cases where the photocatalyst was doped with metals such as silver [19] or upon doping with highly electronegative dopants, such as fluorine [20]. It was claimed

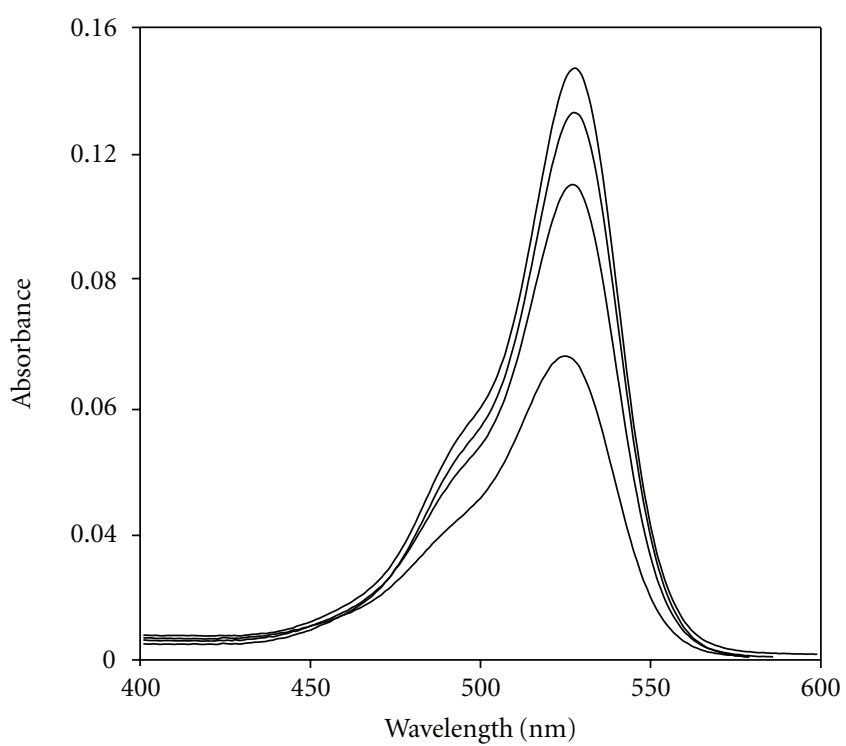

(a)

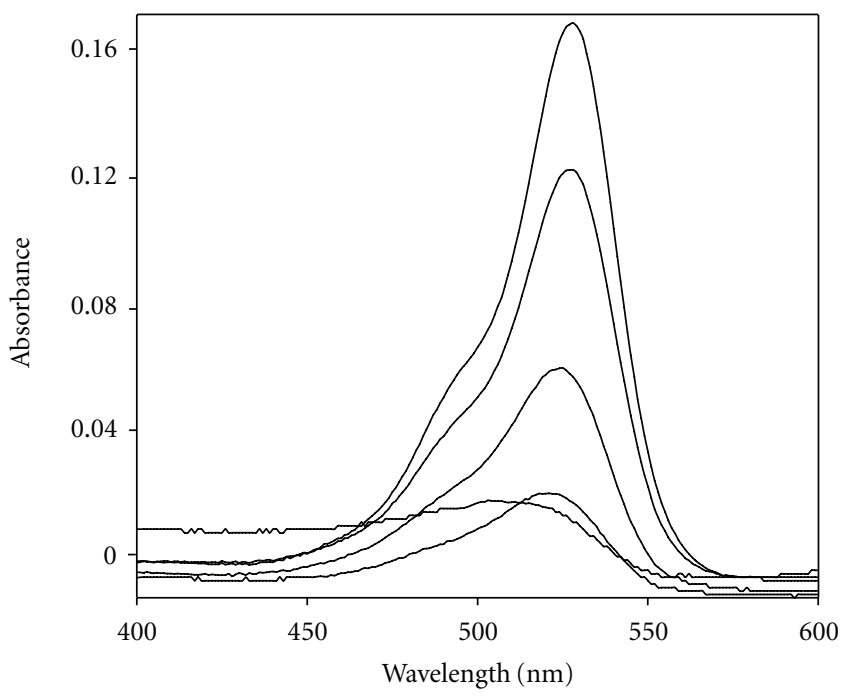

(b)

FIGURE 3: Visible spectra of rhodamine 6G during photocatalytic degradation. (a) Without bias (open-circuit conditions). Exposure times are $0,6,24$, and $70 \mathrm{hrs}$. (b) Under positive bias of $200 \mathrm{mV}$. Exposure times are $0,4,25,50$, and $72 \mathrm{hrs}$.

that breaking up of the chromophore occurs preferentially when the dye (RB) is adsorbed through its carboxyl group, whereas $\mathrm{N}$-deethylation occurs preferentially when the dye is adsorbed through its positively charged $\mathrm{N}^{+} \mathrm{Et}_{2}$ group, which interacts with the negatively charged fluorines. Others [19] attributed N-dealkylation to surface reaction, while chromophore degradation is attributed to liquid-phase reaction. $\mathrm{N}$-deethylation was reported also in $\mathrm{TiO}_{2} / \mathrm{SiO}_{2}$ composites, where silica ( $\mathrm{PZC}$ at $\mathrm{pH} 2$ ) served as the negative site for the positively charged amine group adsorption [21].

Hence, the photocatalytic degradation of R6G, whether under bias or without bias, is characterized by a first step 
involving chromophore degradation, most likely representing adsorption through the carboxyl group of the dye molecule. Such adsorption is likely to be promoted upon positively charging of the $\mathrm{TiO}_{2}$ surface.

The origin of the blue shifting in the R6G spectra at long exposure times under positive bias is not trivial. The distance between the negatively charged platinum electrodes and the positively charged titanium dioxide is by far larger than the few nanometers that appear in the cited-above literature connecting between dealkylation and adsorption of R6G on metallic nanoislands. It is likely therefore that the dealkylation occurs as a second step, once the chromophore is degraded. Optical microscopy examination of the platinum electrodes after excessive use under bias revealed the existence of residues on the platinum electrodes. These residues were not observed in the absence of bias. This suggests that, under positive bias, the platinum electrodes play a role in the photocatalytic process.

Since the photocatalytic degradation of R6G cannot be initialized reductively at the small negative biased potential that was applied to the platinum electrodes or by superoxides formed by reducing dissolved oxygen [22], it is likely that the degradation process begins oxidatively at the $\mathrm{TiO}_{2}$ electrode and the reduction process taking place on the platinum electrode is a secondary process, acting on some intermediate products. Such a mechanism requires that the intermediate products arrive (either by diffusion or convection) from the $\mathrm{TiO}_{2}$ electrode to the platinum electrode. This may point out that the distance between the $\mathrm{TiO}_{2}$ and the platinum electrodes may affect the photocatalytic degradation process.

The lifetime of hydroxyl radicals in water is around $0.3 \mathrm{msec}$, corresponding to a diffusion lengths of $1.7 \mu \mathrm{m}-$ $20 \mu \mathrm{m}[23,24]$ While the diffusion length is well below the distance between the electrodes (up to $100 \mu \mathrm{m}$ ), stirring (which was used in the presented experiments) may easily assist in covering the required distance and may explain a situation where the R6G molecules (positively charged under the experimental condition) are adsorbed on the platinum electrode yet undergo oxidation by diffusion of hydroxyl radicals.

The kinetics of the photocatalytic bleaching was deduced based on the decrease in the absorption peak of the R6G as a function of exposure time and is presented in Figure 4, revealing apparent first-order kinetics. The figure clearly shows that applying a positive bias voltage to the $\mathrm{TiO}_{2}$ electrodes increases the bleaching rate, in comparison with the bleaching rate under open circuit conditions. In that manner, the rate constant under nonbiased short circuit, nonbiased open circuit, a bias of $200 \mathrm{mV}$, and a bias of $400 \mathrm{mV}$ was $0.0078 \mathrm{hr}^{-1}, 0.0095 \mathrm{hr}^{-1}, 0.034 \mathrm{hr}^{-1}$, and $0.041 \mathrm{hr}^{-1}$, respectively. Unlike other pollutants, the increased rate upon biasing is not trivial, taking into account that, under the experimental conditions, the dye molecules are positively charged, hence, are expected to be repelled from the positively charged $\mathrm{TiO}_{2}$ electrodes. The fact that a significant increase in the reaction rate was observed suggests that either the charge separation issue is so crucial that it surpasses the effect of repletion or that the $\mathrm{OH}$ radicals may

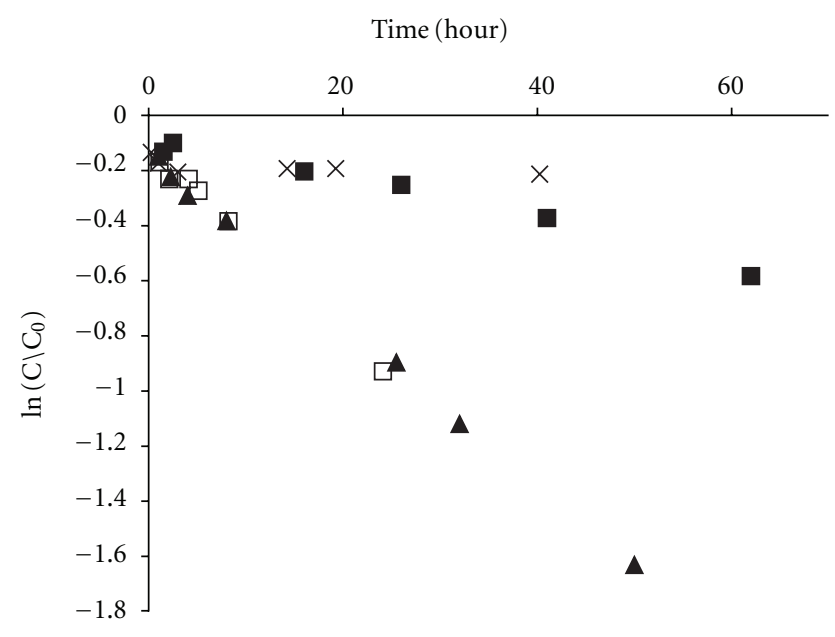

Figure 4: Changes in the concentration of R6G during photodegradation under the following conditions: open circuit (filled squares), short circuit (crosses), and a positive bias of $200 \mathrm{mV}$ (filled triangles), a positive bias of $400 \mathrm{mV}$ (empty squares). The data is based on absorbance measurements at $528 \mathrm{~nm}$.

cover the distance to the negatively charged, R6G coated, platinum electrodes.

The increase in the rate constant between open-circuit conditions and applying $200 \mathrm{mV}$ is significantly larger than the modest increase observed upon increasing the voltage from $200 \mathrm{mV}$ to $400 \mathrm{mV}$. As the voltage is increased, the number of holes at the $\mathrm{TiO}_{2}$ surface becomes closer to its maximum, hence the extent by which activity is enhanced is expected to be reduced. To this one may add the decrease in the tendency of the R6G molecules to be adsorbed or even to be in the vicinity of the $\mathrm{TiO}_{2}$ electrodes.

Using self-assembled monolayers of OCTS chemisorbed on the $\mathrm{TiO}_{2}$ electrode facilitates to decouple between the effect of adsorption and that of polarization. These molecules are known to chemisorb on oxides, and in particular on hydroxylated oxides, such as the titanium dioxide electrode and the silicon dioxide dielectric. Figure 5 presents the C$\mathrm{H}$ stretch envelope in the FTIR spectra of OCTS (i.e., the $\mathrm{CH}_{2}(\mathrm{a}), \mathrm{CH}_{2}(\mathrm{~s}), \mathrm{CH}_{3}(\mathrm{a})$ and $\mathrm{CH}_{3}(\mathrm{~s})$ peaks) prior to exposure to UV, as well as following 5 and 15 minutes of exposure, revealing, as expected, a decrease in the signal. The experiments here were done in air. Previous works with (nonbiased) micrometers size $\mathrm{TiO}_{2}-\mathrm{SiO}_{2}$ stripes onto which self-assembled monolayers had been chemisorbed showed the possibility of remote oxidation by oxidizing species leaving the titanium dioxide surface and attacking molecules chemisorbed on the silica surfaces [13]. In that case, kinetics could be fitted nicely by a two-exponential decaying model, having two characteristic rate constants. The larger rate constant represented the degradation on the photocatalyst's surface, whereas the smaller one represented the degradation on the silica substrate. Following the cited work, the rate constants for the degradation of the molecules chemisorbed on the $\mathrm{TiO}_{2}$ in the present study were calculated based on changes in the integrated absorbance of the $\mathrm{C}-\mathrm{H}$ stretch 


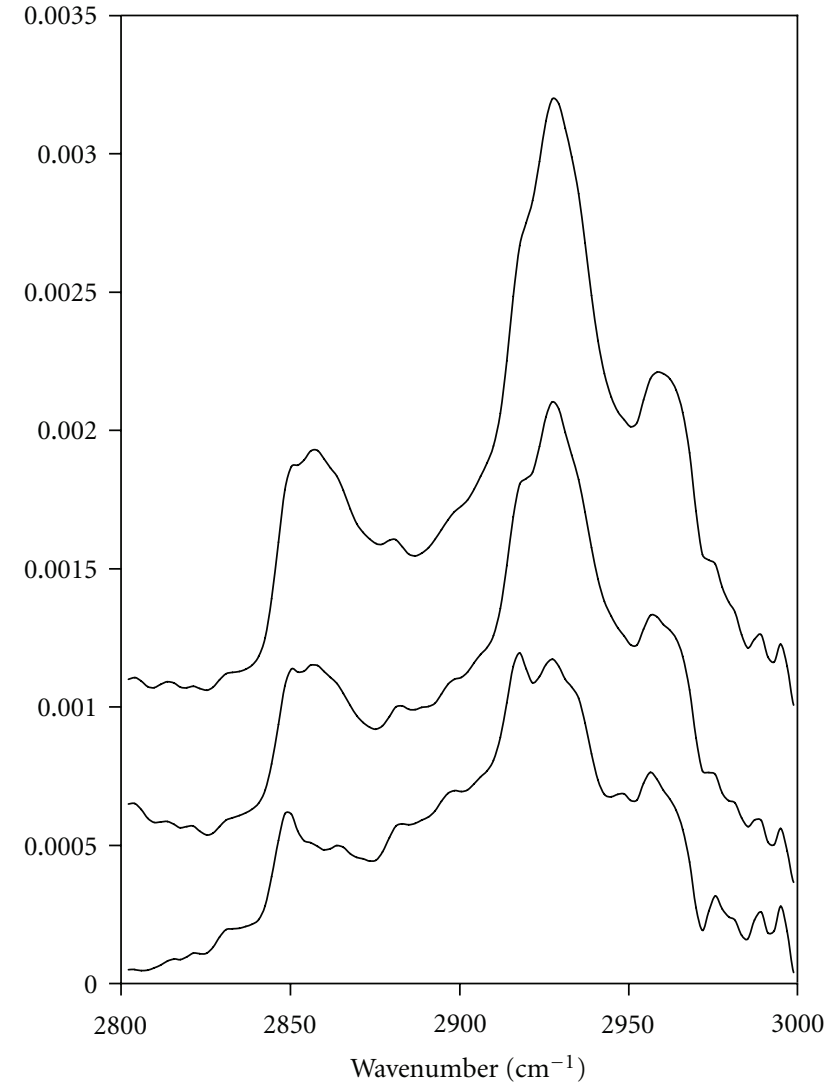

FIGURE 5: FTIR spectra of chemisorbed OCTS prior to exposure to UV light (upper trace) and following $5 \mathrm{~min}$ (middle trace) and $15 \mathrm{~min}$ (lower trace) of exposure under a positive bias of $800 \mathrm{mV}$. The traces were vertically shifted for clarity.

peaks' envelope taking only the data from the first 15 minutes of exposure.

Figure 6 presents the first order rate constants for the degradation of OCTS and their dependence on the electric bias, as measured with two types of electrodes: $50 \mu \mathrm{m}$ $50 \mu \mathrm{m}$ and $100 \mu \mathrm{m}-100 \mu \mathrm{m}$. The figure evidently shows that, for both widths of electrodes, there is an increase in the rate constant as the bias is increased up to $0.4-0.6 \mathrm{~V}$. This increase can be easily rationalized by the decreased rate of recombination induced by biasing positively the photocatalyst. Unexpectedly, as the bias is increased above that level, not only that the rate is no longer increased, but also in fact the oxidation rate of the chemisorbed molecules is significantly reduced. If this phenomenon of counter productive bias had been measured with rhodamine $6 \mathrm{G}$, one could have claimed the reason to be a significant decrease in the adsorption rate of the positively charged R6G. But, here, the OCTS are chemically and irreversibly adsorbed on the electrode, hence the reason has to be different.

A possible explanation could be shortage of superoxide radicals, formed on the platinum electrode, which might be required for the advanced stages in the degradation process of the chemisorbed alkyl chains. As explained below, superoxide radicals, though by themselves ineffective agents

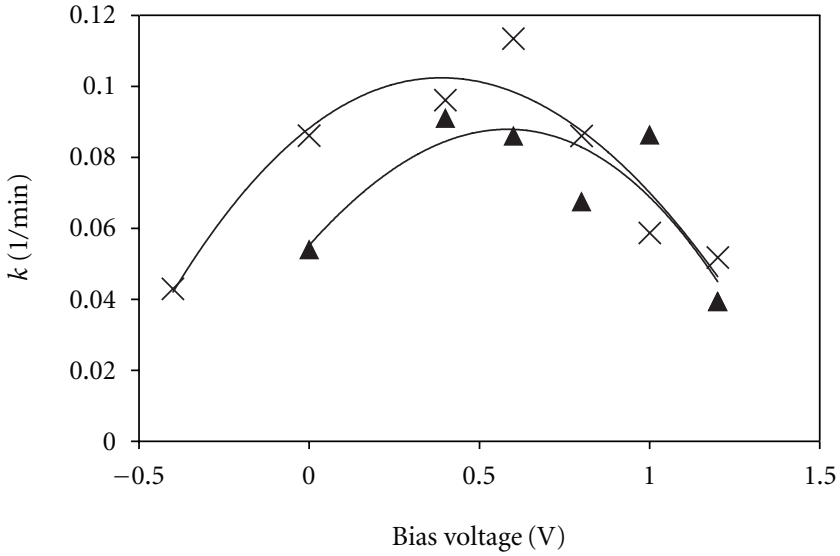

Figure 6: The rate constants measured during the photocatalytic degradation of OCTS as a function of the bias applied to interdigitated electrodes having stripes' width of $100 \mu \mathrm{m}$ (crosses) and $50 \mu \mathrm{m}$ (filled triangles).

for initiating the degradation, may play an important role in the secondary stages of the many photocatalytic processes. That superoxide ions play a role in photocatalytic oxidative processes is well established, for example, by experiments that found that the enzyme superoxide dismutase (SOD) that dismutates superoxide had a detrimental effect on photocatalytic oxidation reactions [25]. This effect was explained by the ability of the superoxide anions to react with the radical cations that are formed upon direct electron transfer from certain pollutants to the valence band of photoexcited titanium dioxide [26]. More evidence came from the work of Schwitzgebel et al. [27] who showed that the degradation path of n-octane, 3-octanol, 3-octanone, and n-octanoic acid went through the formation of organoperoxy radicals (ROO) formed by a primary attack by $\mathrm{OH}$ radicals followed by insertion of molecular oxygen. In these systems, the superoxide radicals combined with the organoperoxy radicals to form unstable tetroxide organoradicals that later decomposed to yield the end products. Within this context, it is noteworthy that the role of back diffusion of reduced oxygen species was demonstrated in the past (in the absence of bias) on surfaces made of alternating stripes of metals $(\mathrm{Pt}, \mathrm{Au})$ and titanium dioxide, onto which self-assembled monolayers were chemisorbed [28]. Hence, one should not rule out the possibility that the reduced rates of degradation observed in the current work (Figure 6) at bias above $0.6 \mathrm{~V}$ stem from shortage in superoxides formed on the Pt electrodes.

We would like to point out that photoelectrochemical disinfection of water containing E. coli showed a monotonic increase in the disinfection rate up to a bias of several Volts, in contrast to our observation $[29,30]$. This lack of counterproductive effect under large bias can be attributed to the fact that bacteria are usually negatively charged (hence adsorption becomes more and more favourable as bias increases) and to the fact that, for disinfection, an attack on the membrane by $\mathrm{OH}$ radicals should be sufficient.

Some words need to be written about the fundamental importance of the small distance between the electrodes 
demonstrated in this work, beyond the specific example of the degradation of R6G. In principle, the distance between the electrodes is not expected to have a major effect on charge separation, since the thickness of the ionic double layer, which partially neutralizes the field between the electrodes, is smaller by far than the distance between the electrodes. Moreover, from the charge separation point of view, the distance between the anode and cathode should not play an important role, since the photocatalytic oxidative reaction is expected to be initiated by holes and/or by oxidized $\mathrm{OH}-$ (i.e., hydroxyl radicals). However, these logics are changed if electrons or reduced dioxygens (superoxide ions) are an integral part of the degradation scheme together with the oxidative agents as indeed happens in many cases.

For these reactions, the presented system can be ideal (in particular once optimization is performed) as it benefits from both low recombination rate and superb ability to utilize the superoxide anions that are required for the secondary stages. In optimizing the system for photocatalysis, one has to take into consideration several factors, among which are the lifetime and diffusion length of $\mathrm{OH}$ radicals, superoxide radicals, and the organoradicals formed upon oxidation by the hydroxyls. As mentioned before, the lifetime of hydroxyl radicals in water was reported to be between $0.3 \mathrm{msec}$ and $1 \mathrm{sec}$, with corresponding diffusion lengths of $1.7 \mu \mathrm{m}-20 \mu \mathrm{m}$. Similarly, the diffusion length of superoxides in water is approximately $10 \mu \mathrm{m}$, with a tendency to decrease upon increasing the $\mathrm{pH}$ [24]. This means that a distance of $10-20 \mu \mathrm{m}$ between the electrodes should be appropriate for conditions where convective mass transport can be neglected. Obviously, this optimal distance is expected to be considerably longer if vigorous mixing takes place.

In conclusion, using interdigitated, planar device made of a photocatalyst and a counter electrode, operating photoelectrocatalytically under small positive bias can be quite beneficial for treating water, even under conditions where the ionic strength is low. The two main benefits are the lack of pressure builtup due to absence of liquid film flow velocity restrictions (recirculating pumping in a batch process or single-pass flow in a free-falling liquid film mode of operation), and, no less important, the ability to promote charge separation while supplying superoxides that in many cases are required for complete mineralization.

\section{Acknowledgments}

The lithographic masks used for preparing the interdigitated electrode arrays were obtained from M. Neumann Spallart (CNRS, Meudon). The help of F. Subl (MetaDesign AG, Berlin) in their preparation is gratefully acknowledged.

\section{References}

[1] P. Salvador, "On the nature of photogenerated radical species active in the oxidative degradation of dissolved pollutants with $\mathrm{TiO}_{2}$ aqueous suspensions: a revision in the light of the electronic structure of adsorbed water," Journal of Physical Chemistry C, vol. 111, no. 45, pp. 17038-17043, 2007.
[2] H. Gerischer and A. Heller, "The role of oxygen in photooxidation of organic molecules on semiconductor particles," Journal of Physical Chemistry, vol. 95, no. 13, pp. 5261-5267, 1991.

[3] V. E. Henrich, G. Dresselhaus, and H. J. Zeiger, "Observation of two-dimensional phases associated with defect states on the surface of TiO2," Physical Review Letters, vol. 36, no. 22, pp. 1335-1339, 1976.

[4] M. R. Hoffmann, S. T. Martin, W. Choi, and D. W. Bahnemann, "Environmental applications of semiconductor photocatalysis," Chemical Reviews, vol. 95, no. 1, pp. 69-96, 1995.

[5] D. W. Bahnemann, J. Mönig, and R. Chapman, "Efficient photocatalysis of the irreversible one-electron and two-electron reduction of halothane on platinized colloidal titanium dioxide in aqueous suspension," Journal of Physical Chemistry, vol. 91, no. 14, pp. 3782-3788, 1987.

[6] N. Shaham-Waldmann and Y. Paz, "Photocatalytic reduction of $\mathrm{cr}(\mathrm{VI})$ by titanium dioxide coupled to functionalized cnts: an example of counter-productive charge separation," Journal of Physical Chemistry C, vol. 114, no. 44, pp. 18946-18952, 2010.

[7] P. Pichat, "Surface properties, activity and selectivity of bifunctional powder photocatalysts," New Journal of Chemistry, vol. 11, pp. 135-140, 1987.

[8] B. Sun, A. V. Vorontsov, and P. G. Smirniotis, "Role of platinum deposited on $\mathrm{TiO}_{2}$ in phenol photocatalytic oxidation," Langmuir, vol. 19, no. 8, pp. 3151-3156, 2003.

[9] S. Kedem, D. Rozen, Y. Cohen, and Y. Paz, "Enhanced stability effect in composite polymeric nanofibers containing titanium dioxide and carbon nanotubes," Journal of Physical Chemistry C, vol. 113, no. 33, pp. 14893-14899, 2009.

[10] L. Wenhua, L. Hong, C. Sao'an, Z. Jianqing, and C. Chunan, "Kinetics of photocatalytic degradation of aniline in water over $\mathrm{TiO}_{2}$ supported on porous nickel," Journal of Photochemistry and Photobiology A, vol. 131, no. 1-3, pp. 125-132, 2000.

[11] G. Waldner, M. Pourmodjib, R. Bauer, and M. NeumannSpallart, "Photoelectrocatalytic degradation of 4-chlorophenol and oxalic acid on titanium dioxide electrodes," Chemosphere, vol. 50, no. 8, pp. 989-998, 2003.

[12] K. Vinodgopal, U. Stafford, K. A. Gray, and P. V. Kamat, "Electrochemically assisted photocatalysis. 2. The role of oxygen and reaction intermediates in the degradation of 4chlorophenol on immobilized $\mathrm{TiO} 2$ particulate films," Journal of Physical Chemistry, vol. 98, no. 27, pp. 6797-6803, 1994.

[13] H. Haick and Y. Paz, "Remote photocatalytic activity as probed by measuring the degradation of self-assembled monolayers anchored near microdomains of titanium dioxide," Journal of Physical Chemistry B, vol. 105, no. 15, pp. 3045-3051, 2001.

[14] E. Zemel, H. Haick, and Y. Paz, "Photocatalytic destruction of organized organic monolayers chemisorbed at the vicinity of titanium dioxide surfaces," Journal of Advanced Oxidation Technologies, vol. 5, pp. 27-32, 2002.

[15] S. Ghosh-Mukerji, H. Haick, M. Schvartzman, and Y. Paz, "Selective photocatalysis by means of molecular recognition," Journal of the American Chemical Society, vol. 123, no. 43, pp. 10776-10777, 2001.

[16] Y. Paz, Z. Luo, L. Rabenberg, and A. Heller, "Photooxidative self-cleaning transparent titanium dioxide films on glass," Journal of Materials Research, vol. 10, no. 11, pp. 2842-2848, 1995.

[17] T. Watanabe, T. Takizawa, and K. Honda, "Photocatalysis through excitation of adsorbates. 1. Highly efficient $\mathrm{N}$ deethylation of rhodamine B adsorbed to CdS," Journal of Physical Chemistry, vol. 81, no. 19, pp. 1845-1851, 1977. 
[18] H. M. Sung-Suh, J. R. Choi, H. J. Hah, S. M. Koo, and Y. C. Bae, "Comparison of Ag deposition effects on the photocatalytic activity of nanoparticulate $\mathrm{TiO}_{2}$ under visible and UV light irradiation," Journal of Photochemistry and Photobiology A, vol. 163, no. 1-2, pp. 37-44, 2004.

[19] M. K. Seery, R. George, P. Floris, and S. C. Pillai, "Silver doped titanium dioxide nanomaterials for enhanced visible light photocatalysis," Journal of Photochemistry and Photobiology A, vol. 189, no. 2-3, pp. 258-263, 2007.

[20] Q. Wang, C. Chen, D. Zhao, W. Ma, and J. Zhao, "Change of adsorption modes of dyes on fluorinated $\mathrm{TiO}_{2}$ and itseffect on photocatalytic degradation of dyesunder visible light," Langmuir, vol. 24, pp. 7338-7345, 2008.

[21] F. Chen, J. Zhao, and H. Hidaka, "Highly selective deethylation of rhodamine B: adsorption and photooxidation pathways of the dye on the TiO2/ SiO2 composite photocatalyst," International Journal of Photoenergy, vol. 5, pp. 209-217, 2003.

[22] N. A. Kuznetsova, L. E. Kilimchuk, and O. L. Kaliya, "Photooxidation of rhodamines," Zhurnal Fizicheskoi Khimii, vol. 66, pp. 2503-2509, 1992.

[23] Y. Kikuchi, K. Sunada, T. Iyoda, K. Hashimoto, and A. Fujishima, "Photocatalytic bactericidal effect of $\mathrm{TiO}$ thin films: dynamic view of the active oxygen species responsible for the effect," Journal of Photochemistry and Photobiology A, vol. 106, no. 1-3, pp. 51-56, 1997.

[24] M. Okuda, T. Tsuruta, and K. Katayama, "Lifetime and diffusion coefficient of active oxygen species generated in $\mathrm{TiO} 2$ sol solutions," Physical Chemistry Chemical Physics, vol. 11, no. 13, pp. 2287-2292, 2009.

[25] P. Pichat, "Some views about indoor air photocatalytic treatment using $\mathrm{TiO}_{2}$ : conceptualization of humidity effects, active oxygen species, problem of C1-C3 carbonyl pollutants," Applied Catalysis B, vol. 99, no. 3-4, pp. 428-434, 2010.

[26] L. Cermenati, P. Pichat, C. Guillard, and A. Albini, "Probing the $\mathrm{TiO} 2$ photocatalytic mechanisms in water purification by use of quinoline, photo-fenton generated $\mathrm{OH}$ radicals and superoxide dismutase," Journal of Physical Chemistry B, vol. 101, no. 14, pp. 2650-2658, 1997.

[27] J. Schwitzgebel, J. G. Ekerdt, H. Gerischer, and A. Heller, "Role of the oxygen molecule and of the photogenerated electron in $\mathrm{TiO}_{2}$-photocatalyzed air oxidation reactions," Journal of Physical Chemistry, vol. 99, no. 15, pp. 5633-5638, 1995.

[28] H. Haick and Y. Paz, "Long-range effects of noble metals on the photocatalytic properties of titanium dioxide," Journal of Physical Chemistry B, vol. 107, no. 10, pp. 2319-2326, 2003.

[29] J. C. Harper, P. A. Christensen, T. A. Egerton, T. P. Curtis, and J. Gunlazuardi, "Effect of catalyst type on the kinetics of the photo electrochemical disinfection of water inoculated with E. coli," Journal of Applied Electrochemistry, vol. 31, no. 6, pp. 623-628, 2001.

[30] N. Baram, D. Starosvetsky, J. Starosvetsky, M. Epshtein, R. Armon, and Y. Ein-Eli, "Enhanced inactivation of E. coli bacteria using immobilized porous $\mathrm{TiO}_{2}$ photoelectrocatalysis," Electrochimica Acta, vol. 54, pp. 3381-3386, 2009. 


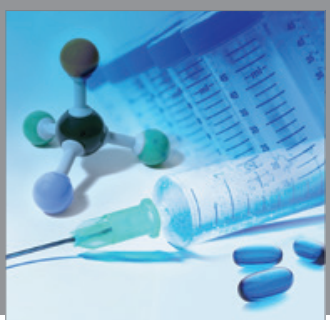

International Journal of

Medicinal Chemistry

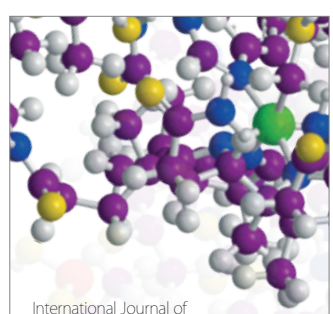

Carbohydrate Chemistry

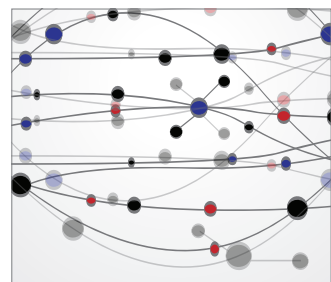

The Scientific World Journal
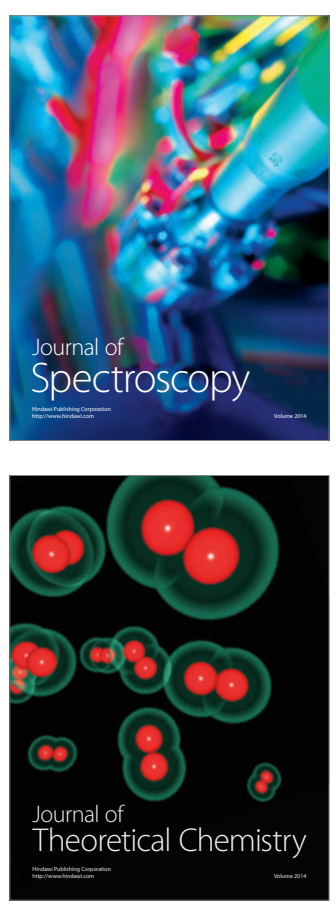
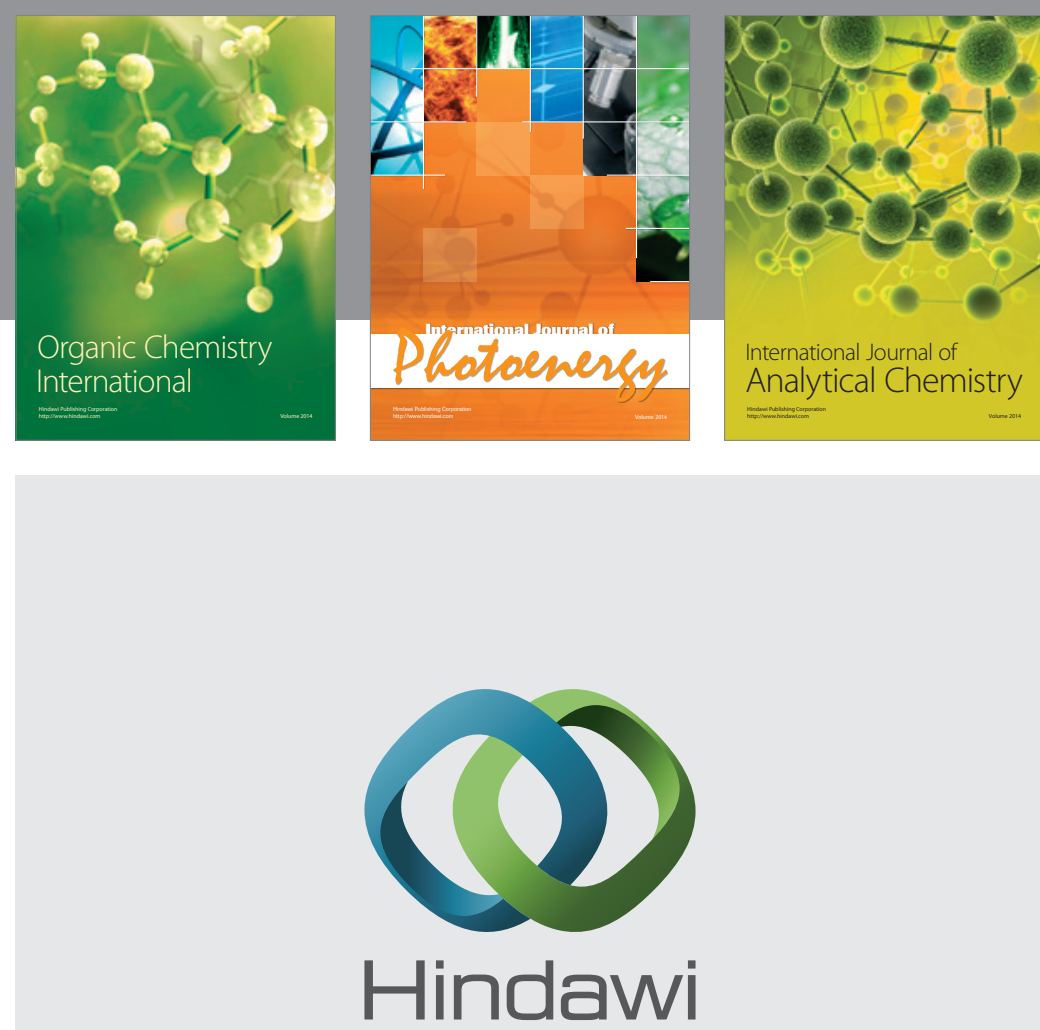

Submit your manuscripts at

http://www.hindawi.com
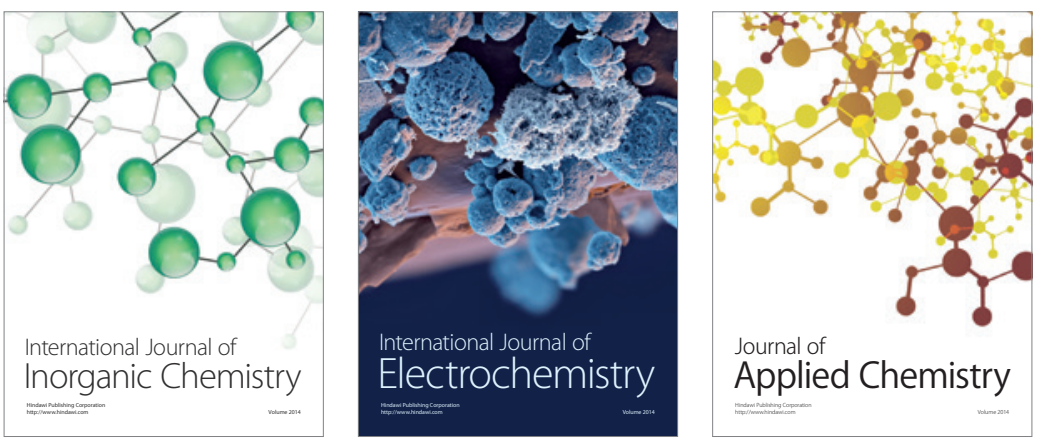

Journal of

Applied Chemistry
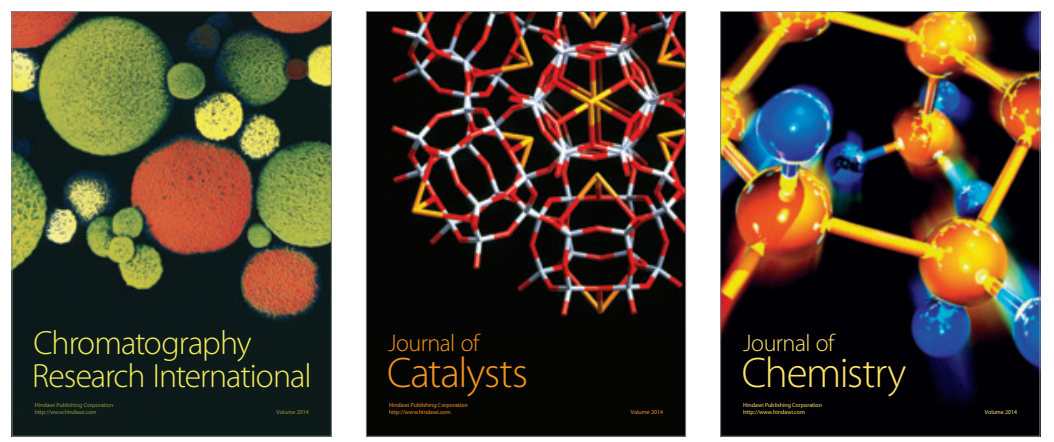
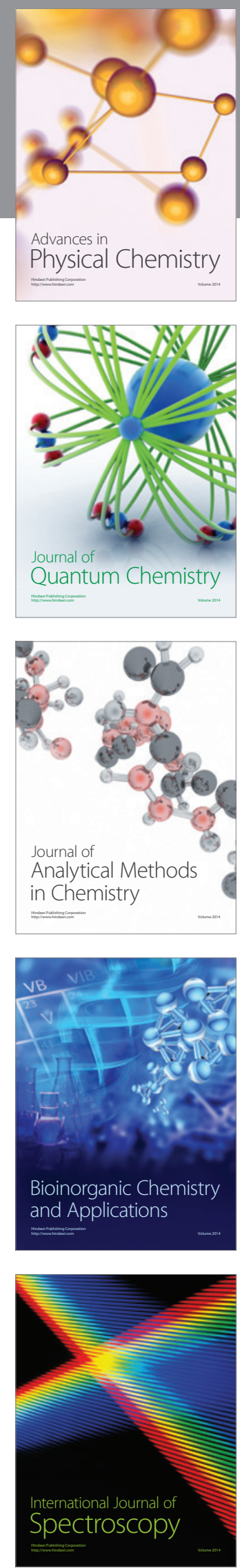